\title{
Exclusive breastfeeding during the 40-day rest period and at six months in Lebanon: a cross-sectional study
}

\author{
Rana F. Chehab', Lara Nasreddine ${ }^{2 *}$, Racha Zgheib ${ }^{3}$ and Michele R. Forman ${ }^{1}$
}

\begin{abstract}
Background: Exclusive breastfeeding is recommended for the first 6 months of life with well-established benefits to the mother and child. The traditional practice of the 40-day rest period helps establish and maintain exclusive breastfeeding. This study aims to estimate the prevalence and examine the factors associated with exclusive breastfeeding at 40 days and at 6 months in Lebanon.

Methods: A cross-sectional survey was conducted in 2011-2012 as part of the "Early Life Nutrition and Health in Lebanon" study. A nationally representative sample of 1005 children aged five years or younger and their mothers was drawn from households using a stratified cluster sampling design. Trained nutritionists interviewed eligible mothers about sociodemographic characteristics of the household and maternal and child characteristics including infant feeding practices. Anthropometric measurements of the mother and child were collected. Multinomial logistic regression analysis was conducted to examine the characteristics associated with exclusive breastfeeding.

Results: The prevalence of exclusive breastfeeding was $41.5 \%$ at 40 days and $12.3 \%$ at 6 months. Children in families with three or more children had higher odds of exclusive breastfeeding for 40 days (Adjusted Odds Ratio [AOR] 1.76, 95\% Confidence Interval [CI] 1.19, 2.60). Children in families owning two or more cars had lower odds of exclusive breastfeeding for 40 days (AOR $0.45,95 \% \mathrm{Cl} 0.24,0.83$ ) and at 6 months (AOR $0.32,95 \% \mathrm{Cl} 0.14,0.77$ ). Similarly, children delivered via Caesarian section had lower odds of exclusive breastfeeding for 40 days (AOR 0.49, $95 \% \mathrm{Cl} 0.34,0.71$ ) and at 6 months (AOR 0.39, 95\% Cl 0.24, 0.65). The odds of exclusive breastfeeding for 6 months were lower among children of overweight (AOR $0.50,95 \% \mathrm{Cl} 0.26,0.95$ ) or obese (AOR $0.56,95 \% \mathrm{Cl} 0.32,0.98$ ) mothers.
\end{abstract}

Conclusions: The association between higher socio-economic status, as reflected by car ownership, and C-section delivery with lower odds of exclusive breastfeeding persisted across the first 6 months in Lebanon. Future research should investigate the factors associated with exclusive breastfeeding in prospective cohort studies and help to better understand the cultural practice of the 40-day rest period in relation to breastfeeding.

Keywords: Exclusive breastfeeding, 40-day rest period, Socio-economic status, C-section delivery, Lebanon

\footnotetext{
* Correspondence: In10@aub.edu.lb

${ }^{2}$ Department of Nutrition and Food Science, American University of Beirut, Beirut, Lebanon

Full list of author information is available at the end of the article
}

(c) The Author(s). 2020 Open Access This article is licensed under a Creative Commons Attribution 4.0 International License, which permits use, sharing, adaptation, distribution and reproduction in any medium or format, as long as you give appropriate credit to the original author(s) and the source, provide a link to the Creative Commons licence, and indicate if changes were made. The images or other third party material in this article are included in the article's Creative Commons licence, unless indicated otherwise in a credit line to the material. If material is not included in the article's Creative Commons licence and your intended use is not permitted by statutory regulation or exceeds the permitted use, you will need to obtain permission directly from the copyright holder. To view a copy of this licence, visit http://creativecommons.org/licenses/by/4.0/ The Creative Commons Public Domain Dedication waiver (http://creativecommons.org/publicdomain/zero/1.0/) applies to the data made available in this article, unless otherwise stated in a credit line to the data. 


\section{Background}

Many societies in the Middle East observe a 40-day postpartum period of rest, seclusion, and ritual that helps establish and maintain breastfeeding $[1,2]$ and protects the mother and newborn from illnesses [3]. During this 40-day rest period, the mother often stays at home and receives help with household chores and congratulatory visits from related women and neighbors [3]. She is encouraged to eat a special diet rich in meat, poultry, soups, and other foods thought to be good for milk production [3]. Other than the cultural aspect, the 40-day rest period has religious underpinnings. For both Muslims and Christians, the 40 days following birth coincide with the period of vaginal discharge resulting from involution of the uterus [4-6]. Despite being closely linked to infant feeding practices, few studies have examined the 40-day rest period in relation to total and exclusive breastfeeding $[1,2]$.

The World Health Organization (WHO) recommends exclusive breastfeeding (EBF) for the first 6 months of life [7]. Despite these recommendations and the wellestablished benefits of breastfeeding, the proportion of mothers who EBF for 6 months in the Middle East is estimated at $20.5 \%$ (95\% CI 14.5, 28.2) [8]. The prevalence of EBF for 6 months in specific countries of the Middle East ranges from 2\% in Kuwait [9] to 56.4\% in Iran [10]. In Lebanon, a small Middle Eastern country on the Mediterranean sea, the prevalence of EBF for 6 months was $10.1 \%$ in a national sample of mother-child dyads recruited from primary healthcare centers in 2004 [11]. More recently in 2016, the prevalence of EBF for 4-6 months was estimated at $16.5 \%$ among mothers of toddlers attending daycare centers [12].

None of the previous studies conducted in Lebanon focused on the 40-day rest period as a cultural practice for breastfeeding. Understanding the context-specific patterns and determinants of breastfeeding practices is essential to ensure successful promotion strategies [13]. Therefore, it is timely to place a lens on the 40-day rest period and to examine the factors associated with EBF in Lebanon. The objectives of this paper are to estimate the prevalence of EBF at 40 days and at 6 months, and to examine the sociodemographic, maternal, and child factors associated with EBF in a nationally representative sample of mother-child dyads in Lebanon in 2011-2012.

\section{Methods}

The study was designed as a cross-sectional survey as part of the "Early Life Nutrition and Health in Lebanon (ELNAHL)" project [14].

\section{Sampling strategy}

A representative sample of children $(N=1194)$ aged 5 years or younger of both sexes was drawn from households using a stratified cluster sampling design. The strata were the six Lebanese governorates and the clusters were selected at the district level. Within each district, households were selected following a probability proportional to size approach, whereby a higher number of participating households was drawn from more populous districts. Housing units constituted the primary sampling unit in the districts of Lebanon.

\section{Eligibility criteria}

Mother-child dyads were eligible to participate in the study if the mother was Lebanese, aged 19-40 years, did not have hypertension or diabetes, and was not taking medications that interfere with eating and breastfeeding or affect body weight. Children were eligible if they were five years old or younger, were born at term (gestational age between 37 and 42 weeks), and had no chronic medical conditions, inborn errors of metabolism, or physical malformations that interfere with feeding patterns and body composition.

\section{Data collection}

Data were collected between September 2011 and August 2012. Trained nutritionists administered the survey through face-to-face interviews with the mothers. The survey inquired about sociodemographic characteristics of the household, current and future family planning practices of the mother, access to maternal and child health services, and mother's knowledge and practices related to infant feeding. Questions on infant feeding practices focused on the duration of total and exclusive breastfeeding, which was assessed using the life-long approach [15], the age of introduction of formula milk and solid food, and the reasons for breastfeeding, not breastfeeding, and breastfeeding cessation. Anthropometric measurements of the mother and child were collected. This study was approved by the Institutional Review Boards at the American University of Beirut (NUT.LN.13) and Purdue University (Protocol number: 1902021663). Mothers provided written informed consents.

\section{Definitions of infant feeding practices}

Infant feeding practices were defined as follows:

1. Exclusive breastfeeding (EBF): The infant received breast milk from his/her mother or expressed breast milk and no other fluids or solids.

2. Mixed feeding: The infant received breast milk with formula milk and/or other fluids and/or solid food.

3. Exclusive bottle feeding (EBOT): The infant received formula milk with or without other fluids. 
4. Bottle and solid feeding (BOT+SF): The infant received formula milk and/or other fluids and solid food.

\section{Statistical analysis}

Frequencies with percentages (\%) and means with standard deviations (SD) were calculated to describe categorical and continuous variables, respectively. Since only 17 mothers $(1.8 \%)$ of the sample were underweight (Body Mass Index $[\mathrm{BMI}]<18.5 \mathrm{~kg} / \mathrm{m}^{2}$ ) at the time of the interview, they were merged with the normal weight mothers $\left(B M I=18.5-24.9 \mathrm{~kg} / \mathrm{m}^{2}\right)$ into one BMI category (normal weight $<25 \mathrm{~kg} / \mathrm{m}^{2}$ ). Chi-squared test and Analysis of Variance (ANOVA) followed by post hoc comparisons (Bonferroni) were calculated, as appropriate, to compare infant feeding practices at 40 days and at 6 months by sociodemographic, maternal, and child characteristics. Multinomial logistic regression models were computed to estimate the adjusted odds ratio (AOR) and 95\% confidence intervals $(\mathrm{CI})$ of EBF compared to mixed feeding and EBOT at 40 days, as well as the odds of EBF compared to mixed feeding and BOT+SF at 6 months. Variables selected for inclusion in the multinomial analysis had a $p$-value $<0.10$ in the bivariate analysis. All statistical analysis was conducted using the Statistical Analysis Package for Social Sciences (SPSS, version 24.0).

\section{Results}

Of the 1194 eligible mother-child dyads that were contacted, 1029 participated in the survey (response rate $86 \%$ ). Twenty-four surveys with missing data were excluded, leaving 1005 mother-child dyads in the analysis. At the time of the interview, 947 children were 40 days or older and had complete information about feeding practices at 40 days, while 893 children were 6 months or older and had complete information about feeding practices at 6 months and thereby included in the analysis at 40 days and at 6 months, respectively.

\section{Prevalence of exclusive breastfeeding and other infant feeding practices at $\mathbf{4 0}$ days and at 6 months}

The prevalence of infant feeding practices at 40 days and at 6 months are presented in Fig. 1. Of the total 1005 children included in the analysis, $89.5 \%$ were ever breastfed. Among those, 27.8\% were first breastfed within $1 \mathrm{~h}$ after delivery, $53.1 \%$ after $1 \mathrm{~h}$ but within the first $24 \mathrm{~h}$, and $19.1 \%$ within days after delivery (data not shown). At 40 days, $41.5 \%$ (95\% CI 38.4, 44.7) of the 947 children were $\mathrm{EBF}, 38.1 \%$ (95\% CI 34.8, 41.2) were mixed fed, and $20.2 \%(95 \%$ CI $17.5,22.7)$ were EBOT. At 6 months, $12.3 \%$ (95\% CI 10.2, 14.6) of the 893 children were EBF, $38.4 \%$ (95\% CI 35.3, 41.7) were mixed fed, and $40.1 \%$ $(95 \%$ CI $36.7,43.3)$ were BOT+SF.

Sociodemographic, maternal, and child characteristics by infant feeding practice at $\mathbf{4 0}$ days and at $\mathbf{6}$ months

Sociodemographic, maternal, and child characteristics by infant feeding practice at 40 days and at 6 months are presented in Table 1. There were significant differences between the infant feeding groups at 40 days in terms of paternal educational level, number of owned cars, number of children in the family, mode of delivery and child's age, weight and height at the time of the interview (overall $p$ - value $<0.05$ ). Specifically, a lower proportion of EBOT children had fathers with an education at an intermediate level or lower compared to mixed fed children (Bonferroni adjusted $p$ - value $=0.008$ [data not shown]). A higher proportion of EBOT children were in

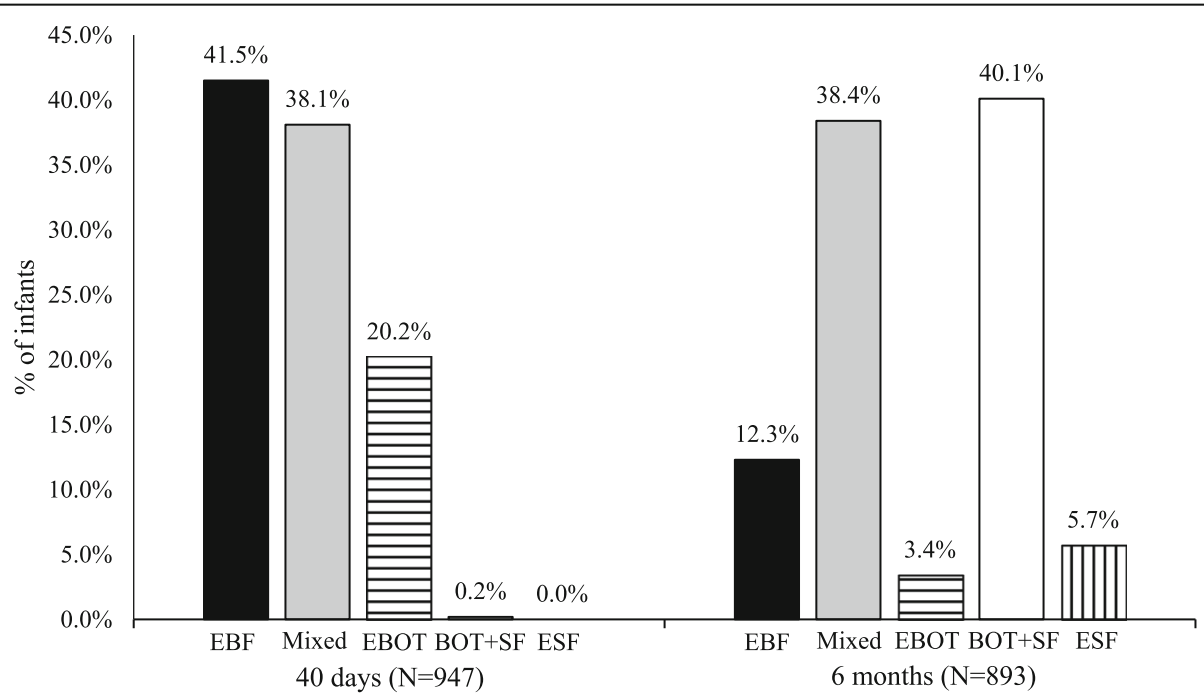

Fig. 1 Prevalence of infant feeding practices at 40 days and 6 months in Lebanon. EBF: Exclusive breastfeeding; EBOT: Exclusive bottle feeding; BOT+SF: Bottle and solid feeding; ESF: Exclusive solid feeding 
Table 1 Sociodemographic, maternal, and child characteristics by infant feeding practice at 40 days and at 6 months in Lebanon

\begin{tabular}{|c|c|c|c|c|c|c|c|c|}
\hline \multirow[t]{2}{*}{ Characteristics ${ }^{\#}$} & \multicolumn{4}{|c|}{ At 40 days $(\boldsymbol{N}=947)$} & \multicolumn{4}{|c|}{ At 6 months $(\boldsymbol{N}=893)$} \\
\hline & $\begin{array}{l}\mathrm{EBF} \\
(\boldsymbol{n}=393)\end{array}$ & $\begin{array}{l}\text { Mixed fed } \\
(\boldsymbol{n}=361)\end{array}$ & $\begin{array}{l}\text { EBOT } \\
(\boldsymbol{n}=191)\end{array}$ & $\begin{array}{l}\boldsymbol{p}- \\
\text { value* }^{*}\end{array}$ & $\begin{array}{l}\mathrm{EBF} \\
(\boldsymbol{n}=110)\end{array}$ & $\begin{array}{l}\text { Mixed fed } \\
(\boldsymbol{n}=343)\end{array}$ & $\begin{array}{l}\mathrm{BOT}+\mathrm{SF} \\
(\boldsymbol{n}=358)\end{array}$ & $\begin{array}{l}\boldsymbol{p}- \\
\text { value* }^{*}\end{array}$ \\
\hline \multicolumn{9}{|l|}{ Sociodemographic characteristics } \\
\hline \multicolumn{9}{|l|}{ Governorate of residence } \\
\hline Beirut & $43(10.9)$ & $31(8.6)$ & $15(7.9)$ & 0.074 & $14(12.7)^{a, b}$ & $31(9.0)^{\mathrm{a}}$ & $33(9.2)^{b}$ & 0.009 \\
\hline Mount Lebanon & $123(31.3)$ & $95(26.3)$ & $67(35.1)$ & & $36(32.7)^{a, b}$ & $83(24.2)^{a}$ & $126(35.2)^{b}$ & \\
\hline North Lebanon & $151(38.4)$ & $134(37.1)$ & $58(30.4)$ & & $37(33.6)^{a, b}$ & $152(44.3)^{a}$ & $107(29.9)^{b}$ & \\
\hline South Lebanon \& Nabatieh & $53(13.5)$ & $67(18.6)$ & $34(17.8)$ & & $17(15.5)^{a, b}$ & $52(15.2)^{a}$ & $64(17.9)^{b}$ & \\
\hline Bekaa & $23(5.9)$ & $34(9.4)$ & $17(8.9)$ & & $6(5.5)^{a, b}$ & $25(7.3)^{a}$ & $28(7.8)^{b}$ & \\
\hline \multicolumn{9}{|l|}{ Family monthly income (USD) } \\
\hline$\leq 400$ & $39(9.9)$ & $38(10.6)$ & $10(5.2)$ & 0.341 & $6(5.5)$ a,b & $49(14.4)^{a}$ & $17(4.8)^{b}$ & $<0.001$ \\
\hline $400.1-1000$ & $180(45.9)$ & $153(42.6)$ & 89 (46.6) & & $56(50.9)^{a, b}$ & $161(47.2)^{a}$ & $157(44.0)^{b}$ & \\
\hline $1000.1-2000$ & $66(16.8)$ & $65(18.1)$ & $47(24.6)$ & & $15(13.6)^{a, b}$ & $49(14.4)^{a}$ & $82(23.0)^{b}$ & \\
\hline$>2000$ & $36(9.2)$ & $30(8.4)$ & $15(7.9)$ & & $13(11.8)^{a, b}$ & $16(4.7)^{\mathrm{a}}$ & $40(11.2)^{b}$ & \\
\hline Doesn't know & $42(10.7)$ & $42(11.7)$ & $16(8.4)$ & & $12(10.9)^{a, b}$ & $44(12.9)^{a}$ & $29(8.1)^{b}$ & \\
\hline Refused to answer & $29(7.4)$ & $31(8.6)$ & $14(7.3)$ & & $8(7.3)^{a, b}$ & $22(6.5)^{a}$ & $32(9.0)^{b}$ & \\
\hline \multicolumn{9}{|l|}{ Paternal educational level } \\
\hline Intermediate or lower & $210(54.7)^{a, b}$ & $205(57.6)^{a}$ & $92(48.2)^{b}$ & 0.035 & $57(52.3)^{a, b}$ & $208(61.7)^{a}$ & $175(49.2)^{b}$ & 0.023 \\
\hline $\begin{array}{l}\text { Secondary education or technical } \\
\text { diploma }\end{array}$ & $113(29.4)^{a, b}$ & $96(27.0)^{a}$ & $76(39.8)^{b}$ & & $35(32.1)^{a, b}$ & $87(25.8)^{a}$ & $123(34.6)^{b}$ & \\
\hline University degree & $61(15.9)^{a, b}$ & $55(15.4)^{a}$ & $23(12.0)^{b}$ & & $17(15.6)^{a, b}$ & $42(12.5)^{a}$ & $58(16.3)^{b}$ & \\
\hline \multicolumn{9}{|l|}{ Paternal employment status } \\
\hline Government or private sector & $229(58.3)$ & $209(57.9)$ & $117(61.3)$ & 0.400 & $67(60.9)$ & $196(57.1)$ & $219(61.2)$ & 0.414 \\
\hline Self-employed & $137(34.9)$ & $136(37.7)$ & $67(35.1)$ & & $34(30.9)$ & $130(37.9)$ & $122(34.1)$ & \\
\hline Unemployed & $27(6.9)$ & $16(4.4)$ & $7(3.7)$ & & $9(8.2)$ & $17(5.0)$ & $17(4.7)$ & \\
\hline \multicolumn{9}{|l|}{ House ownership } \\
\hline Yes & $230(58.5)$ & $215(59.6)$ & $122(63.9)$ & 0.454 & $60(54.5)$ & $206(60.1)$ & $222(62.0)$ & 0.375 \\
\hline No & $163(41.5)$ & $146(40.4)$ & $69(36.1)$ & & $50(45.5)$ & $137(39.9)$ & $136(38.0)$ & \\
\hline \multicolumn{9}{|l|}{ Number of owned cars } \\
\hline 0 & $90(22.9)^{\mathrm{a}}$ & $87(24.1)^{a, b}$ & $36(18.8)^{b}$ & 0.046 & $24(21.8)^{a, b}$ & $96(28.0)^{a}$ & $57(15.9)^{b}$ & $<0.001$ \\
\hline 1 & $235(59.8)^{a}$ & $204(56.5)^{a, b}$ & $102(53.4)^{b}$ & & $66(60.0)^{a, b}$ & $202(58.9)^{a}$ & $198(55.3)^{b}$ & \\
\hline$\geq 2$ & $68(17.3)^{a}$ & $70(19.4)$ a,b & $53(27.7)^{b}$ & & $20(18.2)$ a,b & $45(13.1)^{\mathrm{a}}$ & $103(28.8)^{b}$ & \\
\hline \multicolumn{9}{|l|}{ Crowding index (individuals/room) } \\
\hline$\geq 1$ & $349(88.8)$ & $309(85.6)$ & $163(85.3)$ & 0.334 & $99(90.0)$ & $303(88.3)$ & $301(84.1)$ & 0.138 \\
\hline$<1$ & $44(11.2)$ & $52(14.4)$ & $28(14.7)$ & & $11(10.0)$ & $40(11.7)$ & $57(15.9)$ & \\
\hline \multicolumn{9}{|l|}{ Number of children } \\
\hline $1-2$ & $199(50.6)^{a}$ & $203(56.2)^{a, b}$ & $127(66.5)^{b}$ & 0.001 & $59(53.6)^{a, b}$ & $162(47.2)^{a}$ & $229(64.0)^{b}$ & $<0.001$ \\
\hline$\geq 3$ & $194(49.4)^{a}$ & $158(43.8)^{a, b}$ & $64(33.5)^{b}$ & & $51(46.4)^{a, b}$ & $181(52.8)^{\mathrm{a}}$ & $129(36.0)^{b}$ & \\
\hline \multicolumn{9}{|l|}{ Maternal characteristics } \\
\hline Age (years) ${ }^{\$}$ & $32.1 \pm 5.7$ & $31.2 \pm 6.6$ & $31.4 \pm 6.6$ & 0.122 & $32.5 \pm 5.9$ & $32.2 \pm 6.3$ & $31.3 \pm 6.4$ & 0.064 \\
\hline \multicolumn{9}{|l|}{ Educational level } \\
\hline Intermediate or lower & $201(51.1)$ & $180(49.9)$ & 79 (41.4) & 0.067 & $52(47.3)^{a, b}$ & $193(56.3)^{a}$ & $150(41.9)^{b}$ & 0.001 \\
\hline $\begin{array}{l}\text { Secondary education or technical } \\
\text { diploma }\end{array}$ & $103(26.2)$ & $106(29.4)$ & $72(37.7)$ & & $30(27.3)^{a, b}$ & $97(28.3)^{\mathrm{a}}$ & $121(33.8)^{b}$ & \\
\hline University degree & $89(22.6)$ & 75 (20.8) & $40(20.9)$ & & $28(25.5)^{a, b}$ & $53(15.5)^{\mathrm{a}}$ & $87(24.3)^{b}$ & \\
\hline
\end{tabular}


Table 1 Sociodemographic, maternal, and child characteristics by infant feeding practice at 40 days and at 6 months in Lebanon (Continued)

\begin{tabular}{|c|c|c|c|c|c|c|c|c|}
\hline \multirow[t]{2}{*}{ Characteristics \# } & \multicolumn{4}{|c|}{ At 40 days $(\boldsymbol{N}=947)$} & \multicolumn{4}{|c|}{ At 6 months $(\boldsymbol{N}=893)$} \\
\hline & $\begin{array}{l}\mathrm{EBF} \\
(\boldsymbol{n}=393)\end{array}$ & $\begin{array}{l}\text { Mixed fed } \\
(\boldsymbol{n}=361)\end{array}$ & $\begin{array}{l}\text { EBOT } \\
(\boldsymbol{n}=191)\end{array}$ & $\begin{array}{l}\boldsymbol{p}- \\
\text { value* }\end{array}$ & $\begin{array}{l}\mathrm{EBF} \\
(\boldsymbol{n}=110)\end{array}$ & $\begin{array}{l}\text { Mixed fed } \\
(\boldsymbol{n}=343)\end{array}$ & $\begin{array}{l}\mathrm{BOT}+\mathrm{SF} \\
(\boldsymbol{n}=358)\end{array}$ & $\begin{array}{l}\boldsymbol{p}- \\
\text { value* }\end{array}$ \\
\hline \multicolumn{9}{|l|}{ Employment status } \\
\hline Employed & $65(16.5)$ & $62(17.2)$ & $40(20.9)$ & 0.404 & $14(12.7)$ & 47 (13.7) & $71(19.8)$ & 0.050 \\
\hline Housewives & $328(83.5)$ & $299(82.8)$ & $151(79.1)$ & & $96(87.3)$ & $296(86.3)$ & $287(80.2)$ & \\
\hline \multicolumn{9}{|l|}{ BMI $\left(\mathrm{kg} / \mathrm{m}^{2}\right)$} \\
\hline Normal weight $(<25)$ & $163(44.4)$ & $137(42.2)$ & $71(40.3)$ & 0.903 & $57(53.3)^{a}$ & $118(37.7)^{b}$ & $148(45.8)^{a, b}$ & 0.024 \\
\hline Overweight (25-29.99) & 117 (31.9) & $108(33.2)$ & $58(33.0)$ & & $29(27.1)^{a}$ & $103(32.9)^{b}$ & $105(32.5)^{a, b}$ & \\
\hline Obese $(\geq 30)$ & $87(23.7)$ & $80(24.6)$ & $47(26.7)$ & & $21(19.6)^{a}$ & $92(29.4)^{b}$ & $70(21.7)^{a, b}$ & \\
\hline \multicolumn{9}{|l|}{ Child characteristics } \\
\hline Age (months) ${ }^{\$}$ & $29.6 \pm 15.6^{a}$ & $25.9 \pm 16.1^{b}$ & $25.2 \pm 16.4^{b}$ & 0.001 & $32.3 \pm 13.7^{a}$ & $28.9 \pm 14.7$ & $27.5 \pm 15.5^{b}$ & 0.014 \\
\hline \multicolumn{9}{|l|}{ Gender } \\
\hline Male & $187(47.6)$ & $192(53.2)$ & $103(53.9)$ & 0.204 & $49(44.5)$ & $174(50.7)$ & $195(54.5)$ & 0.176 \\
\hline Female & $206(52.4)$ & $169(46.8)$ & $88(46.1)$ & & $61(55.5)$ & $169(49.3)$ & $163(45.5)$ & \\
\hline \multicolumn{9}{|l|}{ Mode of delivery } \\
\hline Vaginal & $241(61.5)^{\mathrm{a}}$ & $197(55.0)^{\mathrm{a}}$ & $79(41.4)^{b}$ & $<0.001$ & $74(67.3)^{a}$ & $205(59.9)^{a}$ & $157(44.1)^{b}$ & $<0.001$ \\
\hline C-section & $151(38.5)^{a}$ & $161(45.0)^{a}$ & $112(58.6)^{b}$ & & $36(32.7)^{a}$ & $137(40.1)^{a}$ & $199(55.9)^{b}$ & \\
\hline Birthweight $(\mathrm{g})^{\$}$ & $\begin{array}{l}3218.2 \pm \\
500.9\end{array}$ & $\begin{array}{l}3244.4 \pm \\
562.7\end{array}$ & $\begin{array}{l}3169.7 \pm \\
547.0\end{array}$ & 0.297 & $\begin{array}{l}3293.5 \pm \\
512.0\end{array}$ & $\begin{array}{l}3232.1 \pm \\
540.3\end{array}$ & $\begin{array}{l}3188.6 \pm \\
536.9\end{array}$ & 0.178 \\
\hline Birth length $(\mathrm{cm})^{\$}$ & $50.3 \pm 3.0$ & $50.3 \pm 3.0$ & $50.3 \pm 3.4$ & 0.986 & $50.3 \pm 3.3$ & $50.3 \pm 2.8$ & $50.3 \pm 3.1$ & 0.988 \\
\hline Weight $(\mathrm{kg})^{\$}$ & $13.2 \pm 3.8^{a}$ & $12.5 \pm 4.2^{b}$ & $12.7 \pm 3.9^{a, b}$ & 0.043 & $14.0 \pm 3.5^{\mathrm{a}}$ & $12.9 \pm 3.5^{b}$ & $13.2 \pm 3.5^{\mathrm{a}, \mathrm{b}}$ & 0.018 \\
\hline Height $(\mathrm{cm})^{\$}$ & $87.9 \pm 12.8^{a}$ & $84.8 \pm 14.2^{b}$ & $84.9 \pm 14.3^{b}$ & 0.004 & $90.6 \pm 11.1^{\mathrm{a}}$ & $87.4 \pm 12.0^{b}$ & $87.4 \pm 12.4^{b}$ & 0.035 \\
\hline
\end{tabular}

\# Data are presented as frequency (\%) unless otherwise stated

$\$$ Data are presented as mean \pm SD

* Overall $p$-values compare the three infant feeding groups together and are calculated using chi-squared test for categorical variables and ANOVA for continuous variables

${ }^{a-b}$ Infant feeding practices with different superscripts differ significantly after Bonferroni adjustment $(p-$ value $<0.0167)$

families that owned two or more cars $(p-$ value $=0.013)$ and had less than three children $(p-$ value $<0.001)$ compared to EBF children. In addition, a higher proportion of EBOT children was delivered via Caesarian section (C-section) compared to $\operatorname{EBF}(p$ - value $<0.001)$ and mixed fed $(p-$ value $=0.002)$ children. Exclusive breastfed children were older and taller at the time of the interview than mixed fed $(p-$ value $=0.001$ and 0.002 , respectively) and $\mathrm{EBOT} \mathrm{(} p$ - value $=0.002$ and 0.016 , respectively) children. Moreover, EBF children were heavier at the time of the interview than mixed fed children $(p-$ value $=0.015)$.

At 6 months, there were significant differences between the infant feeding groups in terms of governorate of residence, paternal and maternal education, monthly income, number of owned cars, and number of children in the family (overall $p$ - value $<0.05$ ). The groups also differed in terms of maternal BMI, mode of delivery, and child's age, weight and height at the time of the interview. A lower proportion of $\mathrm{BOT}+\mathrm{SF}$ children were in families that lived in North Lebanon (Bonferroni adjusted $p-$ value $=0.001$ ), had lower monthly income $(p$ - value $<0.001)$, and had fathers and mothers with an education at an intermediate level or lower $(p-$ value $=$ 0.004 and $<0.001$, respectively) compared to mixed fed children. In addition, a higher proportion of BOT+SF children were in families that owned two or more cars $(p$ - value $<0.001)$ and had less than three children $(p$ value $<0.001)$ compared to mixed fed children. A higher proportion of EBF children had mothers with normal weight compared to mixed fed children $(p-$ value $=$ 0.016). A higher proportion of BOT $+\mathrm{SF}$ children was delivered via $C$-section compared to $\operatorname{EBF}(p$ - value < $0.001)$ and mixed fed $(p-$ value $<0.001)$ children. EBF children were older than $\mathrm{BOT}+\mathrm{SF}(p-$ value $=0.002)$, heavier than mixed fed children $(p$-value $=0.005)$, and taller than mixed fed $(p-$ value $=0.013)$ and $\mathrm{BOT}+\mathrm{SF}(p$ - value $=0.011)$ children.

Table 2 presents the results of the multinomial logistic regression analysis of the factors associated with EBF for 40 days. Although certain variables had significant overall and/or Bonferroni adjusted $p$-values in the bivariate 
Table 2 Multinomial logistic regression analysis of factors associated with exclusive breastfeeding for 40 days in Lebanon

\begin{tabular}{|c|c|c|}
\hline \multirow[t]{2}{*}{ Characteristics } & \multicolumn{2}{|l|}{ At 40 days $(\boldsymbol{N}=947)^{\mathbf{a}}$} \\
\hline & EBF vs Mixed feeding ${ }^{1}$ & EBF vs EBOT ${ }^{2}$ \\
\hline \multicolumn{3}{|l|}{ Sociodemographic characteristics } \\
\hline \multicolumn{3}{|l|}{ Governorate of residence } \\
\hline Beirut & 1.00 & 1.00 \\
\hline Mount Lebanon & $1.04(0.59,1.83)$ & $0.89(0.44,1.79)$ \\
\hline North Lebanon & $0.88(0.51,1.54)$ & $1.00(0.49,2.02)$ \\
\hline South Lebanon \& Nabatieh & $0.60(0.33,1.10)$ & $0.64(0.30,1.38)$ \\
\hline Bekaa & $0.54(0.26,1.14)$ & $0.58(0.23,1.43)$ \\
\hline \multicolumn{3}{|l|}{ Paternal educational level } \\
\hline Intermediate or lower & 1.00 & 1.00 \\
\hline Secondary education or technical diploma & $1.11(0.66,1.86)$ & $1.44(0.75,2.76)$ \\
\hline University degree & $1.25(0.85,1.82)$ & $0.86(0.55,1.34)$ \\
\hline \multicolumn{3}{|l|}{ Number of owned cars } \\
\hline 0 & 1.00 & 1.00 \\
\hline 1 & $1.10(0.75,1.60)$ & $0.93(0.57,1.52)$ \\
\hline$\geq 2$ & $0.84(0.50,1.41)$ & $0.45(0.24,0.83)$ \\
\hline \multicolumn{3}{|l|}{ Number of children } \\
\hline $1-2$ & 1.00 & 1.00 \\
\hline$\geq 3$ & $1.23(0.90,1.68)$ & $1.76(1.19,2.60)$ \\
\hline \multicolumn{3}{|l|}{ Maternal characteristics } \\
\hline \multicolumn{3}{|l|}{ Educational level } \\
\hline Intermediate or lower & 1.00 & 1.00 \\
\hline Secondary education or technical diploma & $1.09(0.67,1.76)$ & $1.39(0.77,2.49)$ \\
\hline University degree & $0.85(0.58,1.24)$ & $0.76(0.48,1.19)$ \\
\hline \multicolumn{3}{|l|}{ Child characteristics } \\
\hline Age (months) & $1.00(0.98,1.03)$ & $1.03(0.99,1.06)$ \\
\hline \multicolumn{3}{|l|}{ Mode of delivery } \\
\hline Vaginal & 1.00 & 1.00 \\
\hline C-section & $0.78(0.58,1.06)$ & $0.49(0.34,0.71)$ \\
\hline Weight (kg) & $0.96(0.87,1.07)$ & $0.93(0.83,1.05)$ \\
\hline Height (cm) & $1.03(0.98,1.07)$ & $1.01(0.95,1.06)$ \\
\hline
\end{tabular}

a Data are presented as adjusted odds ratio ( $95 \%$ confidence interval)

1 The reference category is Mixed feeding; ${ }^{2}$ The reference category is EBOT

EBF Exclusive breastfeeding; EBOT Exclusive bottle feeding

analysis, they were not significant after adjusting for all the variables in the model. Children in families owning two or more cars and those delivered via $\mathrm{C}$-section had lower odds of EBF than EBOT at 40 days postpartum (AOR 0.45, 95\% CI 0.24, 0.83 and AOR 0.49, 95\% CI $0.34,0.71$, respectively). On the other hand, children in families with three or more children had higher odds of EBF than EBOT at 40 days (AOR 1.76, 95\% CI 1.19, 2.60).

Table 3 presents the results of the multinomial logistic regression analysis at 6 months. The odds of EBF were half those of mixed feeding at 6 months among children of overweight (AOR 0.50, 95\% CI 0.26, 0.95) or obese (AOR 0.56, 95\% CI 0.32, 0.98) mothers. Children in families owning two or more cars and those delivered via Csection had lower odds of EBF than of BOT+SF at 6 months (AOR 0.32, 95\% CI 0.14, 0.77 and AOR 0.39, 95\% CI 0.24, 0.65, respectively).

\section{Discussion}

To our knowledge, this study is the first in the Middle East to examine the prevalence and predictors of EBF during the 40-day rest period. From a nationally 
Table 3 Multinomial logistic regression analysis of factors associated with exclusive breastfeeding at 6 months in Lebanon

\begin{tabular}{|c|c|c|}
\hline \multirow[t]{2}{*}{ Characteristics } & \multicolumn{2}{|l|}{ At 6 months $(\boldsymbol{N}=893)^{\text {a }}$} \\
\hline & EBF vs Mixed feeding ${ }^{1}$ & $\mathrm{EBF}$ vs $\mathrm{BOT}+\mathrm{SF}^{2}$ \\
\hline \multicolumn{3}{|l|}{ Sociodemographic characteristics } \\
\hline \multicolumn{3}{|l|}{ Governorate of residence } \\
\hline Beirut & 1.00 & 1.00 \\
\hline Mount Lebanon & $1.24(0.55,2.80)$ & $0.81(0.36,1.82)$ \\
\hline North Lebanon & $0.73(0.32,1.70)$ & $0.74(0.32,1.72)$ \\
\hline South Lebanon \& Nabatieh & $1.09(0.44,2.74)$ & $0.73(0.29,1.81)$ \\
\hline Bekaa & $0.77(0.23,2.59)$ & $0.56(0.17,1.85)$ \\
\hline \multicolumn{3}{|l|}{ Family monthly income (USD) } \\
\hline$\leq 400$ & 1.00 & 1.00 \\
\hline $400.1-1000$ & $0.39(0.11,1.47)$ & $2.08(0.51,8.50)$ \\
\hline $1000.1-2000$ & $1.19(0.47,3.02)$ & $1.98(0.80,4.88)$ \\
\hline$>2000$ & $0.75(0.25,2.23)$ & $0.87(0.31,2.45)$ \\
\hline Doesn't know & $1.47(0.44,4.93)$ & $1.86(0.60,5.75)$ \\
\hline Refused to answer & $1.08(0.35,3.27)$ & $2.32(0.77,7.00)$ \\
\hline \multicolumn{3}{|l|}{ Paternal educational level } \\
\hline Intermediate or lower & 1.00 & 1.00 \\
\hline Secondary education or technical diploma & $0.88(0.37,2.12)$ & $1.02(0.43,2.40)$ \\
\hline University degree & $1.16(0.65,2.09)$ & $0.97(0.54,1.74)$ \\
\hline \multicolumn{3}{|l|}{ Number of owned cars } \\
\hline 0 & 1.00 & 1.00 \\
\hline 1 & $0.93(0.51,1.68)$ & $0.66(0.35,1.23)$ \\
\hline$\geq 2$ & $1.01(0.42,2.43)$ & $0.32(0.14,0.77)$ \\
\hline \multicolumn{3}{|l|}{ Number of children } \\
\hline $1-2$ & 1.00 & 1.00 \\
\hline$\geq 3$ & $0.85(0.50,1.45)$ & $1.18(0.69,2.02)$ \\
\hline \multicolumn{3}{|l|}{ Maternal characteristics } \\
\hline Age (years) & $1.01(0.97,1.05)$ & $1.03(0.98,1.07)$ \\
\hline \multicolumn{3}{|l|}{ Educational level } \\
\hline Intermediate or lower & 1.00 & 1.00 \\
\hline Secondary education or technical diploma & $1.46(0.66,3.24)$ & $1.73(0.80,3.78)$ \\
\hline University degree & $0.89(0.48,1.64)$ & $1.03(0.56,1.90)$ \\
\hline \multicolumn{3}{|l|}{ Employment status } \\
\hline Employed & 1.00 & 1.00 \\
\hline Housewives & $1.70(0.82,3.53)$ & $1.63(0.80,3.30)$ \\
\hline \multicolumn{3}{|l|}{ BMI $\left(\mathrm{kg} / \mathrm{m}^{2}\right)$} \\
\hline Normal weight $(<25)$ & 1.00 & 1.00 \\
\hline Overweight (25-29.99) & $0.50(0.26,0.95)$ & $0.70(0.36,1.35)$ \\
\hline Obese $(\geq 30)$ & $0.56(0.32,0.98)$ & $0.60(0.34,1.05)$ \\
\hline \multicolumn{3}{|l|}{ Child characteristics } \\
\hline Age (months) & $0.99(0.95,1.04)$ & $1.01(0.96,1.05)$ \\
\hline \multicolumn{3}{|l|}{ Mode of delivery } \\
\hline Vaginal & 1.00 & 1.00 \\
\hline C-section & $0.67(0.40,1.11)$ & $0.39(0.24,0.65)$ \\
\hline
\end{tabular}


Table 3 Multinomial logistic regression analysis of factors associated with exclusive breastfeeding at 6 months in Lebanon (Continued)

\begin{tabular}{clr}
\hline Characteristics & At 6 months $(\boldsymbol{N}=893)^{\mathbf{a}}$ & \multicolumn{1}{c}{ EBF vs BOT+SF ${ }^{\mathbf{2}}$} \\
\cline { 2 - 3 } & EBF vs Mixed feeding ${ }^{\mathbf{1}}$ & $1.06(0.90,1.26)$ \\
\hline Weight $(\mathrm{kg})$ & $1.17(0.98,1.39)$ & $1.00(0.92,1.08)$ \\
Height $(\mathrm{cm})$ & $1.00(0.92,1.08)$ & $1.08)$ \\
\hline
\end{tabular}

a Data are presented as adjusted odds ratio ( $95 \%$ confidence interval)

${ }^{1}$ The reference category is Mixed feeding; ${ }^{2}$ The reference category is BOT+SF EBF Exclusive breastfeeding; $B O T+S F$ Bottle and solid feeding

representative survey in Lebanon, we report a prevalence of EBF of $41.5 \%$ at 40 days and $12.3 \%$ at 6 months.

While belonging to a family with more children was positively associated with EBF at 40 days, belonging to a family owning more cars or being born via C-section was associated with lower odds of EBF at 40 days and at 6 months. In addition, the odds of EBF for 6 months were lower among children whose mothers were overweight or obese.

The 40-day rest period is practiced in several cultures worldwide including the Amazon [16], Greece [4], China [17], Malaysia [18], Nepal [6], India [5], Burma [19], Turkey [20], Negev Bedouins [1, 3], and Egypt [2]. However, few studies have examined the prevalence of total and exclusive breastfeeding during this period $[1,2]$. In a study of Negev Bedouin Arab women [1], 24\% of the women exclusively breastfed their infants for the first 2 months regardless of whether women received help during the first 40 days. In a qualitative study of maternal beliefs about breastfeeding in a poor urban neighborhood in Egypt [2], women reported exclusively breastfeeding their infants for the first 40 days, after which they supplemented the breastmilk with fluids and foods to promote growth and fatness and to decrease the time spent breastfeeding. In Lebanon, the prevalence of EBF for $8-12$ weeks was $27.4 \%$ among a sample of first-time mothers residing in the capital, Beirut, as part of a randomized trial of postpartum depression [21]. The lower rate of EBF in that study compared to ours (41.5\%) might be due to the longer duration at which EBF was assessed (56-84 days), the sample of women representing an urban population in which EBF rates have been suggested to be lower [22, 23], and the selective nature of participating in a postpartum depression study.

The prevalence of EBF for 6 months in our study was estimated at $12.3 \%$ in 2012 . The figure is slightly higher than that estimated by Batal et al. in 2004 (10.1\%) among a national sample of women recruited from health centers operated by the Ministry of Social Affairs [11]. A more recent study by Mattar et al. in 2016 estimated the prevalence of EBF for 4-6 months at 16.5\% among mothers with 12-36-month-old toddlers recruited from a representative sample of licensed daycare centers by the Lebanese Ministry of Public Health [12].
Compared to other countries in the Middle East, our prevalence estimate of EBF for 6 months is closest to that of $12.2 \%$ in Saudi Arabia [24]. Despite the fact that the Levant countries are in geographic proximity and share similar traditions and population characteristics, the rates of EBF for 6 months in Lebanon were only similar to those in Syria at 12.9\% [25], but lower than those in the Gaza Strip in Palestine (24.4\%) [26] and higher than those in Jordan (1\%) [27].

A higher number of cars owned in a household, an indicator of higher socio-economic status, was associated with lower odds of EBF at 40 days and at 6 months. Indeed, the number of cars was correlated with other socio-economic variables including mother's and father's educational level, family monthly income, house ownership, and crowding index (data not shown). Unlike in high-income countries where breastfeeding rates are higher among wealthier and more educated women, breastfeeding rates are lower and the duration is shorter among wealthier women in low- and middle-income countries [28]. According to two cross-sectional studies conducted in middle-income countries, the first in Nigeria in 2012 [29] and the second in Morocco in 2016 [30], mothers of higher socio-economic status had a lower likelihood of EBF for 6 months. Lebanon, a middle-income country, followed a similar trend.

Compared to vaginal delivery, C-section delivery was consistently associated with lower odds of EBF at 40 days and at 6 months. Other studies from Lebanon yielded mixed results on the association between mode of delivery and exclusive breastfeeding. While Mattar et al. [12] found that C-section delivery was associated with shorter duration of exclusive breastfeeding, Batal et al. [11] did not. However, the latter found differences in hospital practices that support or hinder breastfeeding initiation, which in turn affect breastfeeding exclusivity and duration [31]. For example, a smaller proportion of women who delivered via $\mathrm{C}$-section reported that the hospitals discussed the benefits of breastfeeding with them, allowed 24-h rooming-in, and brought the baby for night feeds compared to women who delivered vaginally. Indeed, 24-h rooming-in and bringing the baby often to the mother for feeding were associated with higher odds of breastfeeding initiation within few hours 
after birth. Rates of C-section delivery are high in Lebanon with an estimated prevalence of $49 \%$ among a sample of women who delivered between 2000 and 2015 [32]. This prevalence greatly exceeds the rate for Csection deliveries of $10-15 \%$ suggested by the WHO [33]. Caesarian section delivery along with the associated maternal and newborn complications have been reported to hinder skin-to-skin contact after birth and delay breastfeeding initiation which in turn reduce breastfeeding duration $[34,35]$.

The number of children in the family was associated with EBF for 40 days, where mothers with a larger number of children were more likely to EBF. In a prospective cohort study conducted in six low- and middle-income countries in 2010 [36], nulliparity was associated with lower odds of EBF for the first 42 days postpartum in two of the included countries, Guatemala and Pakistan.

Overweight and obese mothers were less likely to EBF for 6 months. Given the cross-sectional nature of the study, we cannot determine the direction of the association, notably whether mothers who were presumably overweight or obese in pregnancy were less likely to EBF or whether mothers who did not EBF were more likely to be overweight or obese at the time of the interview. Evidence from the literature supports both directions of association between maternal BMI and duration of EBF. In a cross-sectional study in Germany [37], EBF duration was shorter among obese mothers than among normalweight mothers. In an analysis of CDC Pregnancy Risk Assessment Monitoring System of 19,145 mothers from 2004 to 2008 [38], overweight and obese mothers, compared to the normal weight, had higher odds of discontinuing breastfeeding before 6 months due to insufficient milk and breastfeeding difficulties. On the other hand, shorter breastfeeding duration has been implicated in maternal overweight and obesity. In a study of 212 women in Finland in 2007 who were surveyed 16-20 years postpartum [39], mothers who breastfed for less than 6 months had higher total body fat mass and fat mass percentage than mothers who breastfed for more than 6 months.

This study has a number of limitations. First, it is a cross-sectional study with unclear chronology of the factors associated with exclusive breastfeeding. Second, mothers reported infant feeding practices of their children, with potential for more recall bias among mothers of older children due to a longer time since delivery; however, almost half of the children were younger than 2 years of age.

\section{Conclusions}

The study offers a unique lens into infant feeding practices related to the 40-day rest period and first 6 months of life in Lebanon. The prevalence of EBF at 40 days and at 6 months was 41.5 and $12.3 \%$, respectively. The inverse association of higher socio-economic status, as reflected by the number of cars owned, and C-section delivery with lower odds of EBF persisted across the first 6 months. Future research should investigate the factors associated with EBF in prospective cohort studies and help to better understand the cultural practice of the 40day rest period in relation to breastfeeding. Such research can guide effective planning for interventions to improve breastfeeding practices and ultimately children's health status.

\section{Abbreviations}

EBF: Exclusive breastfeeding; EBOT: Exclusive bottle feeding; BOT+SF: Bottle and solid feeding; ESF: Exclusive solid feeding; ANOVA: Analysis of variance; AOR: Adjusted odds ratio; Cl: Confidence interval; BMI: Body mass index

\section{Acknowledgments}

The authors would like to thank Mrs. Fatima Al Zahraa Chokor for reviewing and helping with the statistical analysis.

Ethical approval and consent to participate

This study was approved by the Institutional Review Boards at the American University of Beirut and Purdue University. Mothers provided written informed consents.

\section{Authors' contributions}

R.F.C. conducted the statistical analysis and drafted the manuscript. L.N. was responsible for the conceptualization of the study objectives and methodology and critically reviewed the manuscript. R.Z. contributed to the data collection and critically reviewed the manuscript. M.R.F. provided valuable input for analysis, data interpretation and write-up of the manuscript.

\section{Funding}

This research was funded by Lebanese National Council for Scientific Research (Beirut, Lebanon) through its support of the Associated Research Unit (ARU) on 'Nutrition and Noncommunicable Diseases in Lebanon', and by the University Research Board (American University of Beirut, Lebanon) (Grant number 102724).

\section{Availability of data and materials}

The dataset analyzed during the current study are available from the corresponding author on reasonable request.

\section{Consent for publication}

Not applicable.

\section{Competing interests}

The authors declare that they have no competing interests.

\section{Author details}

${ }^{1}$ Department of Nutrition Science, Purdue University, West Lafayette, IN 47906, USA. ${ }^{2}$ Department of Nutrition and Food Science, American University of Beirut, Beirut, Lebanon. ${ }^{3}$ Inserm 1256 NGERE, Nancy, France.

Received: 1 November 2019 Accepted: 11 May 2020

Published online: 19 May 2020

\section{References}

1. Forman MR, Hundt GL, Towne D, Graubard B, Sullivan B, Berendes HW, et al. The forty-day rest period and infant feeding practices among Negev Bedouin Arab women in Israel. Med Anthropol. 1990;12(2):207-16.

2. Harrison GG, Zaghloul ZS, Galal OM, Gabr A. Breastfeeding and weaning in a poor urban neighborhood in Cairo, Egypt: maternal beliefs and perceptions. Soc Sci Med. 1993;36(8):1063-9. 
3. Hundt GL, Beckerleg S, Kassem F, Abu Jafar AM, Belmaker I, Abu Saad K, et al. Women's health custom made: building on the 40 days postpartum for Arab women. Health Care Women Int. 2000;21(6):529-42.

4. Eastman N, Russell K. Eastman's expectant motherhood. 6th ed. New York: Little; 1977.

5. Bandyopadhyay M. Impact of ritual pollution on lactation and breastfeeding practices in rural West Bengal, India. Int Breastfeed J. 2009;4:2.

6. Sharma S, van Teijlingen E, Hundley $V$, Angell C, Simkhada P. Dirty and 40 days in the wilderness: eliciting childbirth and postnatal cultural practices and beliefs in Nepal. BMC Pregnancy Childbirth. 2016;16:147.

7. World Health Organization. The optimal duration of exclusive breastfeeding: a systematic review. Geneva: World Health Organization; 2001.

8. Alzaheb RA. A review of the factors associated with the timely initiation of breastfeeding and exclusive breastfeeding in the Middle East. Clin Med Insights Pediatr. 2017;11:1179556517748912.

9. Dashti M, Scott JA, Edwards CA, Al-Sughayer M. Predictors of breastfeeding duration among women in Kuwait: results of a prospective cohort study. Nutrients. 2014;6(2):711-28.

10. Vafaee A, Khabazkhoob M, Moradi A, Najafpoor A. Prevalence of exclusive breastfeeding during the first six months of life and its determinant factors on the referring children to the health centers in Mashhad, northeast of Iran-2007. J Appl Sci. 2010;10(4):343-8.

11. Batal M, Boulghourjian C, Abdallah A, Afifi R. Breastfeeding and feeding practices of infants in a developing country: a national survey in Lebanon. Public Health Nutr. 2006:9(3):313-9.

12. Mattar L, Hobeika M, Zeidan RK, Salameh P, Issa C. Determinants of exclusive and mixed breastfeeding durations and risk of recurrent illnesses in toddlers attending day care programs across Lebanon. J Pediatr Nurs. 2019;45:e24.

13. Newton ER. The epidemiology of breastfeeding. Clin Obstet Gynecol. 2004 47(3):613-23.

14. Nasreddine L, Hwalla N, Saliba A, Akl C, Naja F. Prevalence and correlates of preschool overweight and obesity amidst the nutrition transition: findings from a national cross-sectional study in Lebanon. Nutrients. 2017:9(3):266.

15. Greiner T. Exclusive breastfeeding: measurement and indicators. Int Breastfeed J. 2014:9:18.

16. Piperata BA. Forty days and forty nights: a biocultural perspective on postpartum practices in the Amazon. Soc Sci Med. 2008;67(7):1094-103.

17. Liu N, Mao L, Sun X, Liu L, Chen B, Ding Q. Postpartum practices of puerperal women and their influencing factors in three regions of Hubei, China. BMC Public Health. 2006;6:274

18. Fadzil F, Shamsuddin K, Wan Puteh SE. Traditional postpartum practices among Malaysian mothers: a review. J Altern Complement Med. 2016;22(7): 503-8.

19. Sein KK. Beliefs and practices surrounding postpartum period among Myanmar women. Midwifery. 2013;29(11):1257-63.

20. Geçkil E, Şahin T, Ege E. Traditional postpartum practices of women and infants and the factors influencing such practices in south eastern Turkey. Midwifery. 2009;25(1):62-71.

21. Hamade H, Chaaya M, Saliba M, Chaaban R, Osman H. Determinants of exclusive breastfeeding in an urban population of primiparas in Lebanon: a cross-sectional study. BMC Public Health. 2013;13:702.

22. Thu HN, Eriksson B, Khanh TT, Petzold M, Bondjers G, Kim CN, et al. Breastfeeding practices in urban and rural Vietnam. BMC Public Health. 2012;12:964

23. Qiu L, Zhao Y, Binns CW, Lee AH, Xie X. Initiation of breastfeeding and prevalence of exclusive breastfeeding at hospital discharge in urban, suburban and rural areas of Zhejiang China. Int Breastfeed J. 2009:4:1.

24. Amin T, Hablas $\mathrm{H}, \mathrm{Al}$ Qader AA. Determinants of initiation and exclusivity of breastfeeding in Al Hassa, Saudi Arabia. Breastfeed Med. 2011;6(2):59-68.

25. Al-Akour NA, Okour A, Aldebes RT. Factors associated with exclusive breastfeeding practices among mothers in Syria: a cross-sectional study. J Adv Med Med Res. 2014:2713-24.

26. El Kishawi RR, Soo KL, Abed YA, Muda WAMW. Assessment of exclusive breastfeeding practice among Palestinian mothers in the Gaza strip: a mixed methods design. Asian J Med Health. 2018:1-13.

27. Abuidhail J, Al-Modallal H, Yousif R, Almresi N. Exclusive breast feeding (EBF) in Jordan: prevalence, duration, practices, and barriers. Midwifery. 2014;30(3): $331-7$.
28. Victora CG, Bahl R, Barros AJ, França GV, Horton S, Krasevec J, et al. Breastfeeding in the 21st century: epidemiology, mechanisms, and lifelong effect. Lancet. 2016;387(10017):475-90.

29. Onah S, Osuorah DI, Ebenebe J, Ezechukwu C, Ekwochi U, Ndukwu I. Infant feeding practices and maternal socio-demographic factors that influence practice of exclusive breastfeeding among mothers in Nnewi south-East Nigeria: a cross-sectional and analytical study. Int Breastfeed J. 2014;9:6.

30. Habibi M, Laamiri FZ, Aguenaou H, Doukkali L, Mrabet M, Barkat A. The impact of maternal socio-demographic characteristics on breastfeeding knowledge and practices: an experience from Casablanca, Morocco. Int J Pediatr Adolesc Med. 2018:5(2):39-48.

31. Batal M, Boulghaurjian C. Breastfeeding initiation and duration in Lebanon: are the hospitals "mother friendly"? J Pediatr Nurs. 2005;20(1):53-9.

32. Zgheib SM, Kacim M, Kostev K. Prevalence of and risk factors associated with cesarean section in Lebanon - a retrospective study based on a sample of 29,270 women. Women Birth. 2017;30(6):e265-e71.

33. World Health Organization. WHO statement on caesarean section rates. 2015. Report No.: WHO/RHR/15.02.

34. Hobbs AJ, Mannion CA, McDonald SW, Brockway M, Tough SC. The impact of caesarean section on breastfeeding initiation, duration and difficulties in the first four months postpartum. BMC Pregnancy Childbirth. 2016;16:90.

35. Takahashi K, Ganchimeg T, Ota E, Vogel JP, Souza JP, Laopaiboon M, et al. Prevalence of early initiation of breastfeeding and determinants of delayed initiation of breastfeeding: secondary analysis of the WHO global survey. Sci Rep. 2017;7:44868.

36. Patel A, Bucher S, Pusdekar Y, Esamai F, Krebs NF, Goudar SS, et al. Rates and determinants of early initiation of breastfeeding and exclusive breast feeding at 42 days postnatal in six low and middle-income countries: a prospective cohort study. Reprod Health. 2015;12(2):S10.

37. Grube M, Keitel-Korndorfer A, Bergmann S, Wendt V, von Klitzing K, Petroff D. Breastfeeding in obese versus normal-weight German mothers of various socioeconomic status. J Hum Lact. 2016;32(3):546-50.

38. Kair LR, Colaizy $\Pi$. When breast milk alone is not enough: barriers to breastfeeding continuation among overweight and obese mothers. J Hum Lact. 2016:32(2):250-7.

39. Wiklund $P, X u$ L, Lyytikainen A, Saltevo J, Wang Q, Volgyi E, et al. Prolonged breast-feeding protects mothers from later-life obesity and related cardiometabolic disorders. Public Health Nutr. 2012;15(1):67-74.

\section{Publisher's Note}

Springer Nature remains neutral with regard to jurisdictional claims in published maps and institutional affiliations.

Ready to submit your research? Choose BMC and benefit from:

- fast, convenient online submission

- thorough peer review by experienced researchers in your field

- rapid publication on acceptance

- support for research data, including large and complex data types

- gold Open Access which fosters wider collaboration and increased citations

- maximum visibility for your research: over $100 \mathrm{M}$ website views per year

At $\mathrm{BMC}$, research is always in progress.

Learn more biomedcentral.com/submissions 